\title{
Novel Biological Activity of the Region (106-126) on Human Prion Sequence
}

\author{
Naganori Numao, ${ }^{*, a}$ Norihisa Noguchi, ${ }^{b}$ Yukihiro Eguchi, ${ }^{c}$ Satoshi Watanabe, ${ }^{d}$ Tetsuya FukuI, ${ }^{d}$ \\ Tomoyuki Kamino, ${ }^{e}$ Noriko Shimozono, ${ }^{e}$ Akiko YamazaKi, ${ }^{e}$ Susumu Kobayashi, ${ }^{e}$ and \\ Masanori SASATSU ${ }^{b}$ \\ ${ }^{a}$ BioFrontier Institute Inc.; 5-4-21 Nishi-hashimoto, Sagamihara, Kanagawa 229-1131, Japan: ${ }^{b}$ Tokyo University of \\ Pharmacy and Life Science; 1432-1 Horinouchi, Hachioji, Tokyo 192-0392, Japan: ${ }^{c}$ Mitsui Knowledge Industry Co., Ltd.; \\ 2-7-14 Higashinakano, Nakano-ku, Tokyo 164-8555, Japan: ${ }^{d}$ Hoshi University; 2-4-41 Ebara, Shinagawa-ku, Tokyo \\ 142-8501, Japan: and ${ }^{e}$ Faculty of Pharmaceutical Sciences, Science University of Tokyo; Ichigaya Funagawara-machi, \\ Shinjuku-ku, Tokyo 162-0826, Japan. Received August 7, 2002; accepted November 6, 2002
}

\begin{abstract}
We report that the synthetic peptide Prp106-126 (KTNMKHMAGAAAAGAVVGGLG-COOH) and the reversed peptide Prp126-106 (GLGGVVAGAAAAGAMHKMNTK-COOH) of human prion (hPrp) can express the decarboxylase activity for oxaloacetate in the presence of trifluoroethanol, similar to that of Oxaldie 1 (LAKLLKALAKLLKK-CONH ${ }_{2}$ ) reported previously. The degree of the relative activity of Prp106-126 and Prp126-106 to Oxaldie 1 is 0.47 and 0.21 , respectively. Based on this experimental result, we applied the informational system method (ISM) developed by Veljkovic et al. to the amino acid sequence of Prp106-126 and Prp126-106 to extract a common factor. The same spectra were obtained, indicating that the same periodicity may be conserved on their sequences, as a necessary factor for expressing the same biological activity, irrespective of the orientation of the primary sequence.
\end{abstract}

Key words prion; oxaloacetate; decarboxylation; synthetic peptide; bioinformatics

In the previous study we proposed an empirical method ${ }^{1)}$ which could predict the active site of various proteins $(e . g$, receptors, oncoproteins, cytokines, hormones) from the amino acid sequences. As an application of the method, we synthesized the naturally occurring peptide and the reversed peptide that is an opposite amino to carboxy orientation (vice versa), and examined the biological activity. We indicated the possibility that both peptides express the similar biological function, though their stereochemistry is completely different. ${ }^{2)}$ Recently, we reported that MSI-78A (GIGKFLKKAKKFAKAFVKILKK-CONH ${ }_{2}$ ) and the reversed peptide A87ISM (KKLIKVFAKAFKKAKKLFKGIG-CONH ${ }_{2}$ ) showed the same decarboxylase activity for oxaloacetate to give pyruvate and carbon dioxide in aqueous trifluoroethanol (TFE) ${ }^{3)}$ similar to that of the peptide Oxaldie 1 (LAKLLKALAKLLKK-CONH${ }_{2}$ ) and the reversed peptide 1 Eidlxao (KKLLKALAKLLKAL-CONH${ }_{2}$ ). In this, Oxaldie 1 has been rationally synthesized to express the activity. ${ }^{4)}$ A common factor of MSI-78A and Oxaldie 1 tends to form the amphipathically $\alpha$-helical polycationic structure necessary for expressing the activity in aqueous TFE solution. ${ }^{3)}$

Human prion protein, composed of 253 amino acid residues, is host-encoded and exists in at least two forms. One is referred to as normal prion protein $\left(\operatorname{Prp}^{C}\right)$ the function of which in the cell is still poorly understood, while it has been suggested that the prion protein may play a physiological role in skeletal muscle. The other is known as $\operatorname{Prp}^{\mathrm{SC}}$ (for scrapie), which is an infectious form and has become a worldwide problem. ${ }^{5,6)}$ Thus, the prion-related diseases have been generally accepted as a group of fatal neurodegenerative disorders associated with the misfolding of prion protein.

The recent analysis of the three-dimension structure of $\operatorname{Prp}^{\mathrm{C}}$ by NMR has indicated that the N-terminal segment $23-120$ is flexibly disordered, and that the three regions (amino acid numbers 144-154, 173-194, 200-228) are the $\alpha$-helices, ${ }^{7)}$ while the $\alpha$-helical structure around the four regions $(109-122,129-141,178-191,202-218)$ had already been proposed by various secondary structure prediction methods. ${ }^{8)}$ Owing to the former result, ${ }^{7)}$ less attention and effort are given to test a potential activity of the region $109-122$ predicting the $\alpha$-helical structure, though the association of the region around Prp106-126 (KTNMKHMAGAAAAGAVVGGLG-COOH) with an infective neurotoxic $\operatorname{Prp}^{\mathrm{SC}}$ molecule has been intensively studied. ${ }^{9,10)}$

In the course of visualizing the secondary structure of the region of Prp109-122 (MKHMAGAAAAGAVV) (PDB: 1QM1) by the wheel-plot feature as described previously, ${ }^{3)}$ we noticed that the region Prp106-126 can be formed as an amphiphilical $\alpha$-structure; the two hydrophilic lysines except asparagine are condensed on one side, and the hydrophobic groups of methionine, valine or histidine are on the other (Fig. 1). The wheel-plot feature, in a broad sense, could be regarded as the amphipathically $\alpha$-helical structure. Therefore, it seems worthwhile to examine whether the decarboxy-

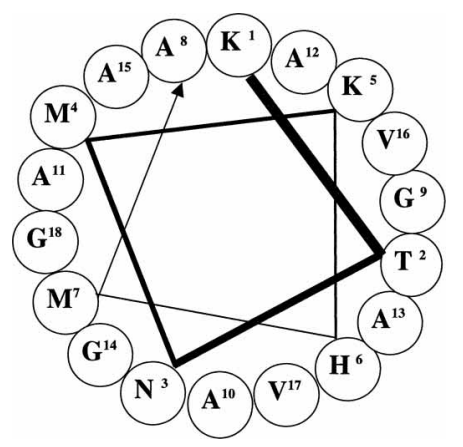

Fig. 1. Wheel-Plot Feature of Prp106-126

One letter represents amino acid residues on Prp106-126. Amino acid residues are numbered from $\mathrm{N}$ - to C-terminal direction, and three residues (GLG) of C-terminal region are not plotted on the feature. 
lase activity of Prp106-126 exists in aqueous TFE solution at $\mathrm{pH}$ 7.0.

In this study, we report the decarboxylase activity of the peptide (Prp106-126) and the reversed peptide Prp126106 (GLGGVVAGAAAAGAMHKMNTK-COOH) for oxaloacetate in the presence of TFE. Furthermore, we have applied the informational system method (ISM) originally developed by Veljkovic et al. ${ }^{11)}$ to the amino acid sequence of the normal and the reversed peptide, in order to extract a common factor other than an electrostatic or a steric one, necessary for expressing the same activity from the primary sequences.

\section{MATERIALS AND METHODS}

Materials The peptides (Oxaldie1, Prp106-126, Prp126-106) and the N-terminal acetyl derivative of Prp106-126 (Ac-KTNMKHMAGAAAAGAVVGGLG$\mathrm{COOH}$; Ac-Prp106-126) used in this study were purchased from TAKARA BIO INC. Japan. In order to analyze various amino acid sequences by a digital processing method (or ISM), we independently constructed the program based on a series of Veljkovic's and related publications, ${ }^{11-16)}$ while the term ISM has been renamed RRM (resonant recognition method). ${ }^{14)}$ Microsoft Excel and Basic are used as programming language, and OS is Windows PC. In brief, the sidechain of 20 kinds of amino acids of a protein sequence was replaced with the electron-ion interaction potential (EIIP) value. ${ }^{12)}$ The value is essentially derived from general-model pseudopotential in the metal ions, ${ }^{15}$ ) while the physicochemical property of EIIP is yet unsolved. Then, the numerical sequences obtained were subjected to discrete fourier transform (DFT) analysis to give the spectra. Finally, we examined the cross-spectrum between ACP-FS (Escherichia coli) and ACP-CL (Krebsiella aerogenes) to give the same spectra as that reported by Lazovic, ${ }^{16}$ ) in order to verify the establishment of our program.

Decarboxylation Assay According to the previous procedure, ${ }^{3)}$ we determined the loss of absorbance at $285 \mathrm{~nm}$ (Ultraspec 3100 pro, Amersham Biosciences Corp.) arising from the enol form of oxaloacetate. In brief, to $1.4 \mathrm{ml}$ (or $1.6 \mathrm{ml}$ ) of the buffer solution ( $50 \mathrm{~mm}$ MOPS, $0.15 \mathrm{M} \mathrm{NaCl}$, $\mathrm{pH}$ 7.0) in the presence (or absence) of $0.2 \mathrm{ml}$ TFE was added $2 \mathrm{~mm}$ sample $(0.2 \mathrm{ml})$. The mixture was stirred at room temperature for $48 \mathrm{~h}$. After stirring, $0.2 \mathrm{ml}$ of oxaloacetate was added to give totally $2.0 \mathrm{ml}$, and this was stirred for $1 \mathrm{~min}$ at room temperature. Then, the reducing absorbance of $285 \mathrm{~nm}$ for $5000 \mathrm{~s}$ was measured. The relative activity of Prp106-126 related peptides was computed by the ratio of their activity of them in the presence (a) or absence (b) of $10 \%$ TFE to the activity of Oxaldie 1 in the presence (c) or absence (d) of $10 \%$ TFE: relative activity $=a-b / c-d$. Each assay was performed in duplicate.

\section{RESULTS AND DISCUSSION}

The aim of this study was to show a third example of the naturally occurring synthetic peptide and the reversed peptide with the same biological activity, prior to developing a new bioinformatics which may elucidate a specific proteinprotein interaction from the primary sequence.
Table 1. The Relative Decarboxylase Activity of Prp106-126 RelatedPeptides to Oxaldie 1 in the Presence of $10 \%$ TFE

\begin{tabular}{lccc}
\hline \hline \multicolumn{1}{c}{ Peptide } & $10 \%$ TFE & $\Delta$ O.D. $^{a}$ & Relative activity \\
\hline Oxaldie 1 & + & 0.267 & 1 \\
Prp106-126 & - & 0.114 & \\
& + & 0.177 & 0.47 \\
Prp126-106 & - & 0.105 & 0.21 \\
& + & 0.143 & \\
Ac-Prp106-126 & - & 0.111 & 0.74 \\
& + & 0.213 & \\
& - & 0.1 &
\end{tabular}

a) $\Delta$ O.D; each number indicates the difference of absorbance at $285 \mathrm{~nm}$ between the starting point $(0 \mathrm{~s})$ and end point $(5000 \mathrm{~s})$ of the reaction.

The decarboxylase activity of the peptide Prp106-126 and the reversed peptide Prp126-106 for oxaloacetate was observed in the presence of $10 \%$ TFE. The activity was enhanced in the case of the peptide Ac-Prp106-126, which is the N-terminal acetyl derivative of Prp106-126, indicating that the reaction is caused by the amino group of the sidechain of either lysine or both on the amino acid sequence. However, their activity was fairly suppressed in the absence of TFE. As shown in Table 1, the relative decarboxylase activity of Prp106-126, Prp126-106 and Ac-Prp106-126 to Oxaldie 1 in the presence of $10 \%$ TFE was $0.47,0.21$ and 0.74 , respectively. The degree of their relative activity is less than that of Oxaldie 1. In addition, the activity of the naturally occurring peptide Prp106-126 was stronger than that of the reversed peptide Prp126-106, similar to the case of Oxaldie 1 and 1 Eidlaxo (or MSI-78A and A87-ISM). Based on the previous study, ${ }^{3)}$ the $\alpha$-helical content of Prp106126 in the presence of TFE may be higher than that of Prp126-106, though their $\alpha$-helical contents in the CD spectrum have not been measured yet. In addition, the reaction may be caused by a great $\mathrm{p} K_{\mathrm{a}}$ shift of the amino group of a lysine side chain on the amphipathically formed $\alpha$-helical structure, ${ }^{4)}$ while it is presently unknown which amino group of the two lysines can participate in the reaction. The great $\mathrm{p} K_{\mathrm{a}}$ shift of the amino group of lysine side chain will be discussed elsewhere using a model compound.

Together the decarboxylase activity of Prp106-126 ordered the secondary structure in the presence of TFE with the cytotoxicity of its intact disordered structure reported previously, ${ }^{17,18)}$ the biological function derived from the region of Prp106-126 of $\operatorname{Prp}^{\mathrm{C}}$ may be regulated in the presence or absence of any auxiliary factor, that is, such as a metal or $\beta$ breaker peptide. ${ }^{19)}$

As a trial to elucidate the biological activity of Prp106126 and Prp126-106 from a new viewpoint as described above, we focused on ISM developed by Veljkovic et al (see Materials and Methods), although so far the homology search and motifs of amino acid sequence, including the stereochemistry have been reported. The ISM is a theoretical method of analysis of the informational content of protein or nucleic acid distribution of valence electrons. In this, they reported that one characteristic frequency could be extracted from at least two or more proteins with the same biological function. In addition, this method does not depend on the degree of homology between the amino acid sequences.

To verify the information, we examined synthetic peptides with the same decarboxylase activity on two points; 1) 
whether a common factor in the analysis of naturally occurring and reversed peptide sequence could be observed, and 2) whether a characteristic frequency could be extracted from the cross-spectra of at least two of the three peptides (Prp106-126, Oxaldie 1 and MSI-78A).

The first, using the same procedure as ISM, we analyzed and contrasted the spectra of Prp106-126 with these of Prp126-106. The result was that the amino acid sequences of Prp106 - 126 and Prp126 - 106 gave the same spectra, irrespective of the orientation (Fig. 2a, 2b). Furthermore, the same spectra were obtained in the case of Oxaldie1 (or MSI78A) and 1 Eidlaxo (or A87-ISM), including TNFR (159178 ) and TNFR (178-159), ${ }^{2)}$ respectively (data not shown). This indicates that the same periodicity based on the transition polarity (i.e., the EIIP value) of amino acid ${ }^{20)}$ is conserved in both the naturally occurring and the reversed sequence, while a strong periodicity of the hydrophobicity has already been reported on the protein primary structure. ${ }^{21)}$ The same result has been obtained in the case of various proteins ${ }^{1)}$ with longer amino acid sequence than that of the peptides used in this study. However, the method may not be sufficient for elucidating the same biological activity, because the reversed $\beta$ amyloid peptide ( $\beta 40-1)$ has no significant neurotoxic activity, unlike that of $\beta$-amyloid peptide $(\beta 1-$ 40). ${ }^{22)}$

The second point, based on the fact that one characteristic frequency could be extracted from a cross-spectrum of at least two or more protein sequences with the same activity, was to extract a characteristic one from at least two of Prp106-126, Oxaldie 1 and MSI-78A (or Prp126-106, 1 Eidlaxo and A87-ISM). As shown in Fig. 3a, one major characteristic frequency $(0.2813)$ could be observed from the

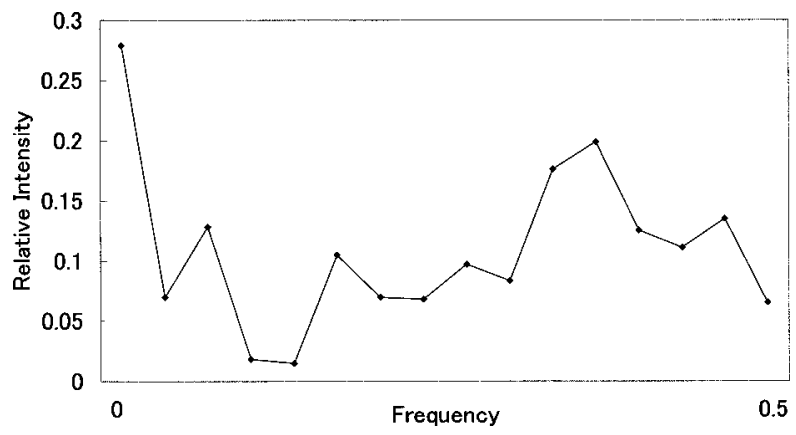

Fig. 2a. The Spectra of Prp106-126

The abscissa represents frequencies from 0.0000 to 0.5000 and the ordinate relative intensities (amplitudes) in the spectra.

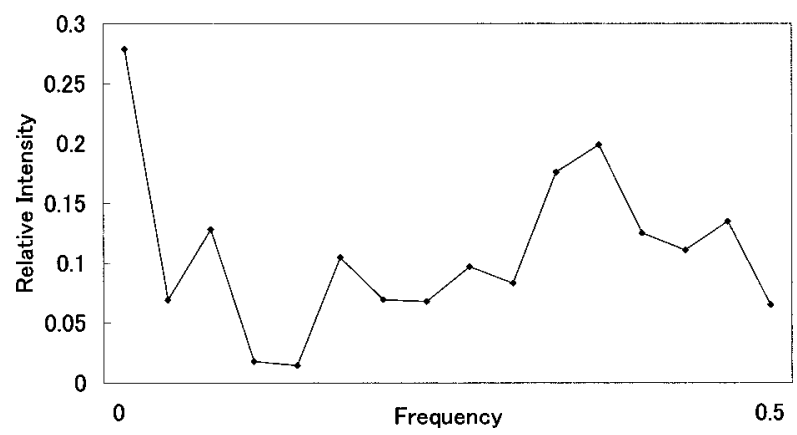

Fig. 2b. The Spectra of Prp126-106

The abscissa and the ordinate represent the same indexes as Fig. 2a. cross-spectrum of all of Prp106-126, Oxaldie 1 and MSI78A. The frequency $(0.2813)$ could be reproduced from the cross-spectrum (Fig. 3b) from Prp106-126 and Oxaldie 1 (or MSI-78A and Oxaldie1), but not from Prp106126 and MSI-78A (Fig. 3c) in spite of the same activity being exerted. Similar results have been observed in other proteins with longer amino acid sequence and the same biological activity (data not shown). Thus, the original ISM is incomplete as a tool to extract a specific frequency from the primary sequence of at least two proteins with the same bioactivity, although the cross-spectrum of various proteins, including acidic and basic fibroblast growth factor, have been reported to show a characteristic frequency. ${ }^{14)}$ We are now in the process of developing a new method to elucidate the same biological activity of Prp106-126, Oxaldie 1 and MSI-78A, including a specific protein-protein interaction, using other

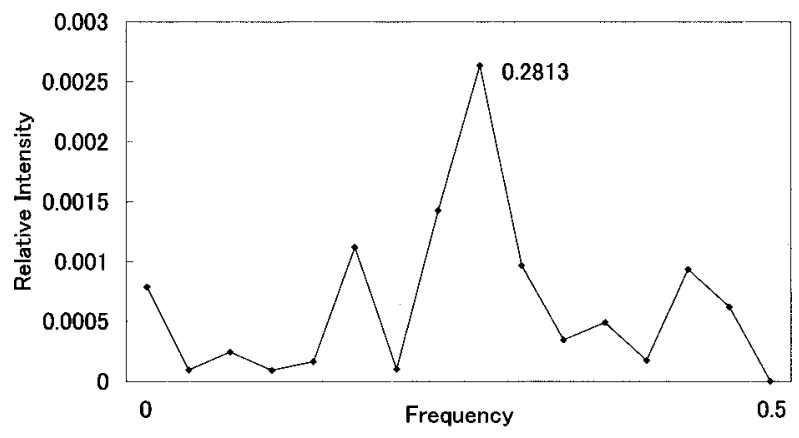

Fig. 3a. The Cross-Spectra of Prp106-126, Oxaldie 1 and MSI-78A

The abscissa and the ordinate represent the same indexes as Fig. 2a. The prominent peaks denote common frequency components.

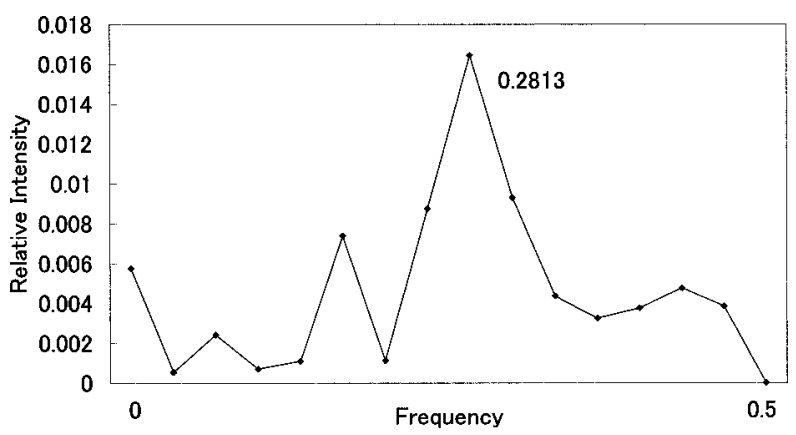

Fig. 3b. The Cross-Spectra of Prp106-126 and Oxaldie 1

The abscissa and the ordinate represent the same indexes as Fig. 2a. The prominent peaks denote common frequency components.

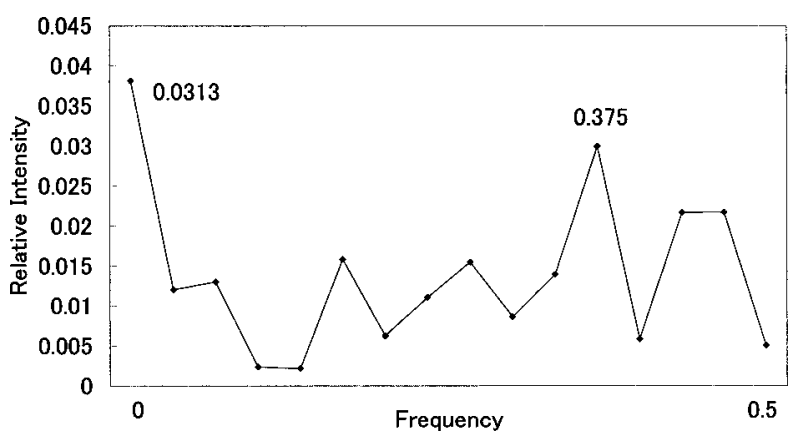

Fig. 3c. The Cross-Spectra of Prp106-126 and MSI-78A

The abscissa and the ordinate represent the same indexes as Fig. 2a. The prominent peaks denote common frequency components. 
physicochemical properties of amino acids.

In conclusion, we have demonstrated that both Prp106126 and the reversed peptide Prp126-106 could express the same decarboxylase activity for oxaloacetate in the presence of TFE, as in the case of Oxaldie 1. This indicates a possibility that both the peptides form $\alpha$-helical structure in the presence of TFE, and then the amino group of lysine side chain on the structure exists in the free form even at $\mathrm{pH} 7.0$ for expressing the decarboxylase activity. Based on this, the in vitro or in vivo decarboxylase activity of human $\operatorname{Prp}^{\mathrm{C}}$ can be expected if the $\alpha$-helical structure on region $106-126$ is inherently viewed as the normal form. Furthermore, the $\operatorname{Prp}^{\mathrm{C}}$ may be physiologically involved in something like the TCA cycle or the biosynthesis of fatty acid. We applied ISM to their amino acid sequences, and the same spectra were obtained for each, irrespective of the orientation of the primary sequence. Based on this finding, although a different degree of the same biological activity cannot be sufficiently explained by this method, the amino acid sequences with the same biological activity may conserve the same or similar periodicity as a necessary factor, indicating that the homology between protein sequences with the same biological function can be represented by the periodicity.

Acknowledgements We very much appreciate Dr. V. Veljkovic at the Institute for Nuclear Science, VINCA, Yugoslavia for his useful comments and suggestions in making a program of ISM, and Professor K. Tanaka at Kyoto University, Japan for critical comments on the physical properties of EIIP value.

\section{REFERENCES}

1) Numao N., Kidokoro S., Biol. Pharm. Bull., 16, 1160-1163 (1993).

2) Lie B.-L., Iwahori A., Sampe R., Hirota Y., Tsubota Y., Yoshioka H.,
Fuchita S., Nakamura N., Saita R., Tunemoto D., Numao N., Biol. Pharm. Bull., 19, 1602-1606 (1996).

3) Numao N., Hirota Y., Iwahori A., Kidokoro S., Sasatsu M., Kondo I., Itoh S., Itoh E., Katoh T., Shimozono N., Yamazaki A., Takao K., Kobayashi S., Biol. Pharm. Bull., 22, 73-76 (1999).

4) Johnsson K., Allemann R. K., Widmer H., Benner S. A., Nature (London), 365, 530-532 (1993).

5) Prusiner S. B., Proc. Natl. Acad. Sci., U.S.A., 95, 13363-13383 (1998) and references therein.

6) Westaway D., Telling G., Priola S., Proc. Natl. Acad. Sci., U.S.A., 95, 11030-11031 (1998) and references therein.

7) Glockshuber R., Hornemann S., Billeter M., Riek R., Wider G., Wuthrich K., FEBS Lett., 426, 291-296 (1998).

8) Huang Z., Gabriel J.-M., Baldwin M. A., Fletterick R. J., Prusiner S. B., Cohen F. E., Proc. Natl. Acad. Sci., U.S.A., 91, 7139-7143 (1994).

9) Tagliavin F., Lievens P. M.-J., Tranchant C., Walter J.-M., Mohr M., Giaccone G., Perini F., Rossi G., Salmona M., Piccardo P., Ghetti B., Beavis R. C., Bugiani O., Frangione B., Prelli F., J. Biol. Chem., 276, 6009-6015 (2001).

10) Kaneko K., Ball H. L., Wille H., Zhang H., Groth D., Torchia M., Tremblay P., Safar J., Prusiner S. B., DeArmond S. J., Baldwin M. A., Cohen F. E., J. Mol. Biol., 295, 997-1007 (2000).

11) Veljkovic V., Cosic I., Dimitrijevic B., Lalovic D., IEEE Transactions on Biomedical Engineering, BME-32, 337-341 (1985).

12) Veljkovic V., Cosic I., Cancer Biochem. Biophys., 9, 139-148 (1987).

13) Cosic I., Nesic D., Eur. J. Biochem., 170, 247-252 (1987).

14) Cosic I., IEEE Transactions on Biomedical Engineering, 41, 11011114 (1993).

15) Veljkovic V., Slavic I., Phys. Rev. Lett., 29, 105-109 (1972).

16) Lazovic J., CABIOS Commun., 12, 553-562 (1996).

17) Forloni G., Angeretti N., Chiesa R., Monzani E., Salmona M., Bugiani O., Tagliavini F., Nature (London), 362, 543-546 (1993).

18) Brown D. R., Schmidt B., Groschup M. H., Kretzschmar H. A., Eur. J. Cell Biol., 75, 29-37 (1998).

19) Soto C., Kascak R. J., Saborio G. P., Aucouturier P., Wisniewski T., Prelli F., Kascsak R., Mendez E., Harris D. A., Ironside J., Tagliavini F., Carp R. I., Frangione B., Lancet, 355, 192-197 (2000).

20) Personal communication about EIIP value from V. Veljkovic.

21) Eisenberg D., Weiss R. M., Terwilliger T. C., Proc. Natl. Acad. Sci., U.S.A., 81, 140-144 (1984).

22) Kowall N. W., Beal M. F., Busciglio J., Duffy L. K., Yankner B. A., Proc. Natl. Acad. Sci., U.S.A., 88, 7247-7251 (1991). 